\title{
Arte mueble paleolítico en el interior peninsular: la cueva de Maltravieso (Cáceres, España)
}

\author{
Palaeolithic portable art in the interior of the Iberian Peninsula: Maltravieso cave \\ (Cáceres, Spain)
}

Marcos García-Díez (*)

Antonio J. Rodríguez Hidalgo (**) $(* * *)(* * * *)$

Antoni Canals Salomó $(* *)(* * *)(* * * *)$

\section{RESUMEN}

Se presenta una pieza ósea grabada con líneas rectilíneas y paralelas recuperada en el nivel A de la Sala de las Chimeneas de la cueva de Maltravieso, datado en $17840 \pm 90 \mathrm{BP}$ y $17930 \pm 100 \mathrm{BP}$. El tema muestra paralelos temáticos con piezas peninsulares y pirenaicas, que abarcan una dilatada cronología a lo largo del Paleolítico superior. La similitud iconográfica y compositiva, y la vinculación espacial del soporte decorado con una parte del arte rupestre de la cavidad, permiten relacionar cronológicamente ambos grafismos y considerarlos como parte integrada de una misma estructura simbólica. Esta evidencia completa un vacío geográfico de arte mueble en el interior peninsular.

\begin{abstract}
We present a bone decorated with engraved straight and parallel lines, recovered in the level I in the Sala de Las Chimeneas at Maltravieso cave, dated at $17840 \pm 90$ $B P$ and $17930 \pm 100$ BP. The motif has similarities with
\end{abstract}

(*) Departamento de Geografía, Prehistoria y Arqueología, Universidad del País Vasco (UPV/EHU). C/ Tomás y Valiente s/n. 01006 Vitoria. Correo e.: diez.garcia.marcos@gmail.com

(**) IPHES, Institut Català de Paleoecología Humana: Evolució Social (Catalonian Institute of Human Paleoecology and Social Evolution). C/ Marcelli Domingo s/n e Campus Sescelades URV (Edifici w3). 43007 Tarragona. España.

Correos e.: arodriguez@iphes.cat; antoni.canals@prehistoria. urv.net

(***) Área de Prehistoria (Department of Prehistory). Universitat Rovira i Virgili (URV). Avinguda de Catalunya 35. 43002 Tarragona. Spain.

$(* * * *)$ EPPEX, Equipo Primeros Pobladores de Extremadura. Casa Municipal de Cultura "Rodríguez Moñino". Avda. Cervantes s/n. 10005 Cáceres, Spain.

Recibido: 4-IV-2011; aceptado: 19-VI-2011. other Peninsula and Pyrenaean evidence Iberian, which has an extensive chronology throughout the Upper Paleolithic. The iconographic and compositional similarities and the spatial relationship with the cave's rock art allow us to relate chronologically both graphics, and interpret them as an integrated part of the same symbolic structure. This evidence fills a geographic gap of portable art in the interior of the Iberian Peninsula.

Palabras clave: Arte prehistórico; análisis técnico y temático; Extremadura; España; Paleolítico superior.

Key words: Prehistoric art; technical and thematic analysis; Extremadura; Spain; Upper Palaeolithic.

\section{INTRODUCCIÓN}

El interior de la Península Ibérica ha sido tradicionalmente caracterizado por una baja ocupación humana durante el Paleolítico Superior, en contraste con el área cantábrica. En los últimos años esta consideración de vacíos geográficos ha cambiado para esta (Delibes y Díez 2006) y otras áreas peninsulares (Mangado 2010).

$\mathrm{El}$ arte rupestre se ha considerado un elemento representativo de la densidad habitacional. Los lugares al aire libre y cavidades identificados, y el alto número de representaciones que contienen muchos de ellos (Alcolea y Balbín 2003; Balbín 2009; Baptista y Reis 2009; Collado 2009), reflejan que el interior de la Península Ibérica fue un lugar intensamente transitado y ocupado por poblaciones humanas con una movilidad elevada (Aubry et al. 2002). 
La práctica ausencia de datos cronológicos precisos en yacimientos arqueológicos implicó que el arte rupestre haya sido utilizado para reconocer la amplitud cronológica de la frecuentación humana, diferenciándose dos posiciones: quienes defienden una ocupación equilibrada y dilatada en el tiempo (Balbín 2009), y quienes proponen conectarla preferentemente con fases más recientes (Corchón 2002). En la actualidad, la progresiva identificación y excavación de nuevos sitios arqueológicos permite considerar, al menos, una significativa y dilatada ocupación humana en este territorio (por ejemplo en Foz Côa, Aubry 2009) desde el Gravetiense.

A pesar de ello, el interior peninsular se ha caracterizado por la práctica ausencia de piezas de arte mueble (Corchón 2002) que precisaran el momento concreto de la actividad gráfica, caracterizaran cada una de las fases y sirvieran para extrapolar las consideraciones hacia los conjuntos parietales. En este contexto de parquedad documental, la pieza de la cueva de Maltravieso aquí presentada y los descubrimientos realizados recientemente en Portugal (García Díez y Aubry 2002; Aubry y Sampaio 2009; Simón et al. 2012) vienen a cubrir este vacío, permitiendo empezar a definir la evolución gráfica paleolítica y encuadrar a partir de bases sólidas la/s fases/s de ejecución de los conjuntos rupestres.

\section{LOCALIZACIÓN}

La cueva de Maltravieso está situada en la ciudad de Cáceres (Extremadura, España) (Fig. 1A). Forma parte del carst de calizas paleozoicas conocido como Calerizo Cacereño (Ripoll López et al. 1999). Su formación está relacionada con un proceso intenso de carstificación estructural originado fundamentalmente por la corrosión desarrollada en favor de los planos de debilidad (diaclasas y planos de estratificación) de la roca carbonatada. En la actualidad la cueva es una forma fósil. Su relleno sedimentario corresponde al aporte de materiales del exterior por varias bocas de entrada relacionadas con cúpulas de disolución distribuidas a lo largo de toda la cueva, y actualmente colmatadas por sedimentos detríticos autóctonos y alóctonos.

Fue descubierta en 1951 durante la explotación de una cantera de caliza (Callejo 1958). En la actualidad presenta un recorrido de $77 \mathrm{~m}$ distribuidos en salas más o menos amplias interconectadas por estrechos corredores (Fig. 1B). Es conocida principalmente por sus motivos de arte rupestre paleolítico distribuidos a lo largo de toda la cavidad. Destacan las representaciones de manos en negativo datadas en el Gravetienese (Almagro-Basch 1960; Breuil 1960; Jordá Cerdá 1971; Jordá Cerdá y Sanchidrián 1992; Ripoll López et al. 1999) que fueron realizadas con ocre mediante aerógrafo. El catálogo actual incluye 71 manos asociadas a pinturas zoomorfas, trianguliformes y puntos, además de grabados de distintas temáticas. La propuesta cronológica para el conjunto artístico es amplia, desde el Auriñaciense medio-final al Solutrense final/Magdaleniense inicial (Ripoll López et al. 1999).

Desde el año 2002 se realizan trabajos arqueológicos. Se ha excavado en el exterior, sin la obtención de resultados arqueológicos contextualizados, en la Sala de los Huesos, donde se ha documentado el uso intenso de la cavidad por parte de carnívoros y un bajo impacto de la actividad humana durante momentos del Pleistoceno medio (Rodríguez-Hidalgo et al. 2010a), y en la
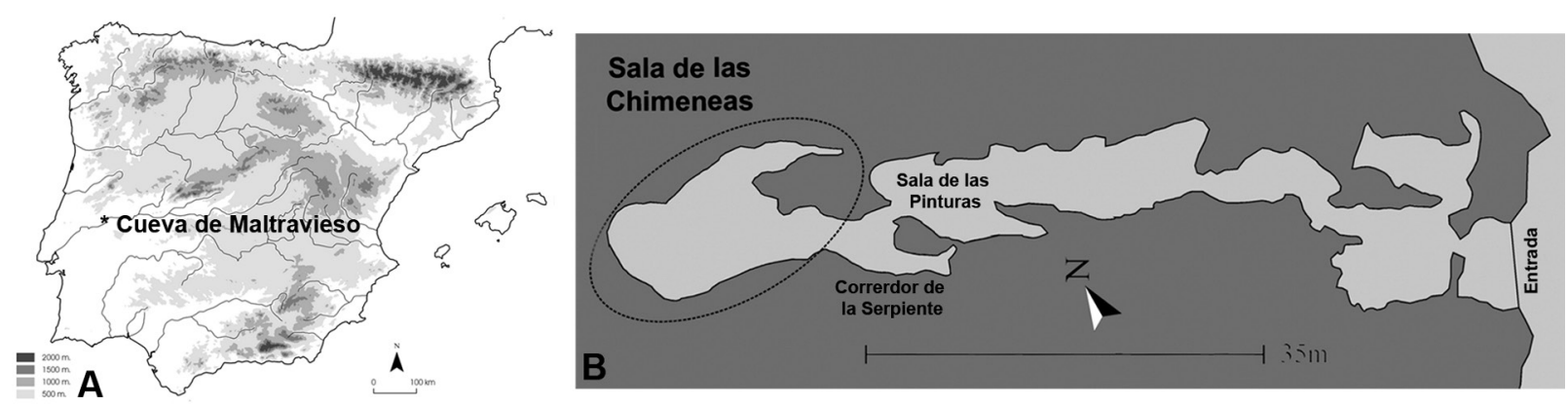

Fig. 1. Cueva de Maltravieso (Cáceres, España). A: localización; B: topografía y situación de la Sala de las Chimeneas.

Trab. Prehist., 69, N. ${ }^{\circ}$, julio-diciembre 2012, pp. 349-356, ISSN: 0082-5638 doi: $10.3989 /$ tp.2012.12096 
Sala de las Chimeneas, donde se han recuperado materiales del Paleolítico superior, como el soporte óseo que se presenta.

El hueso grabado se recuperó en el nivel A de la Sala de las Chimeneas (Canals et al. 2010; Rodríguez-Hidalgo et al. 2011), la última cámara de la cueva, de planta sub-circular, sección oval y con una superficie de más de $150 \mathrm{~m}^{2}$. El nivel A, que no supera los $20 \mathrm{~cm}$ de potencia, se extiende por un área de más de $40 \mathrm{~m}^{2}$. La matriz gruesa (bloques de caliza y cuarzo) está relacionada con un cono de derrubios cuyos materiales proceden del exterior a través de una de las cúpulas de disolución; la fina (arcillas plásticas de descalcificación de color marrón-rojizo) rellena los intersticios entre los bloques.

Los materiales aparecen en la parte más superior del nivel, cubierto por arcillas (nivel superficial) filtradas por las diaclasas, lo que implica que las frecuentaciones humanas acontecieron tras la deposición del nivel A. El conjunto lítico (Canals et al. 2010) lo forman 93 efectivos tallados principalmente sobre cuarzo, aunque también sobre sílex y cuarcita. Predominan las lascas simples $(83 \%)$ seguidas de los fragmentos $(10 \%)$. Los útiles configurados son escasos: una raclette, un raspador carenado, dos muescas y un fragmento con retoque. La talla laminar es escasa. La poca representatividad de este conjunto impide su adscripción tecno-cultural.

Entre la fauna destacan los conejos (Oryctolagus cunniculus) con el 91\% del NISP (Número de Especímenes Identificados). Otros taxones son Equus ferus, Equus hydruntinus, Cervidae indet. Sus scrofa, Ursus arctos, Vulpes vulpes, Lynx pardinus y Felis silvestris. El estudio zooarqueológico indica una variedad de agentes y procesos tafonómicos implicados en su acumulación: destaca la actividad de pequeños carnívoros (zorro y/o lince) y el uso de la cavidad como lugar de captación y procesamiento de conejos por parte de los humanos (Rodríguez-Hidalgo et al. 2011). La malacofauna es escasa: dos conchas perforadas de origen marino (Littorina obtusata y Patella vulgata) utilizadas como elementos de adorno (Rodríguez-Hidalgo et al. 2010b) manifiestan la movilidad de los grupos (a la costa hay una distancia de $300 \mathrm{~km}$.) y/o el intercambio de materiales entre ellos.

Los datos paleoambientales obtenidos del análisis antracológico y de microvertebrados muestran un clima húmedo y de temperatura suave (Bañuls y López-García 2010; Canals et al. 2010).

La valoración del conjunto arqueológico del nivel I evidencia la realización de actividades de subsistencia y merodeo de bajo impacto antrópico en el interior de la sala, centradas en la captura y procesamiento de conejos.

Las dataciones AMS realizadas sobre dos carbones de rama arrojan una edad para el nivel de $17840 \pm 90$ BP (Poz-30469, 21563-20885 cal BP IntCal09 -2 $\sigma-)$ y $17930 \pm 100 \mathrm{BP}(\mathrm{Poz}-30460$, 21758-21041 cal BP IntCal09 -2 $\sigma-)$. Ambas fechas remiten al final del estadio GI2 (2180021200 cal BP) e inicios del GS2c (21000-19000 cal BP), cuando se produce un progresivo enfriamiento climático.

\section{EVIDENCIA GRÁFICA MUEBLE}

El soporte utilizado es un fragmento de diáfisis de costilla de un herbívoro de talla grande (probablemente un bovino) (Fig. 2A y 2B). Presenta fracturas antiguas de tipo diagenético y conserva una de las caras principales de la costilla (medial/ lateral) y uno de los bordes (craneal/caudal). La superficie muestra micro-desconchados provocados por procesos de desecación y corrosión química. Son visibles a partir de 100 aumentos y, si bien han afectado de forma general a la superficie de la pieza y enmascaran algunas características técnicas de las incisiones, no han alterado significativamente el soporte a nivel macroscópico. Las alteraciones post-deposicionales por cambios de humedad de la matriz sedimentaria y por la probable presencia de murcielaguina, afectan a todo el registro óseo del nivel A. Aunque las alteraciones son reconocibles no influyen en la identificación de marcas microscópicas sobre hueso.

El soporte se encuentra fracturado por tres lados, afectando al desarrollo de los surcos. El motivo hoy reconocido debió extenderse por una superficie mayor y formar parte de un diseño más amplio y/o más complejo. Las dimensiones máximas son: $3 \mathrm{~cm}$ de ancho, $1,5 \mathrm{~cm}$ de alto y 0,15 $\mathrm{cm}$ de grosor.

La cara superior presenta una serie de líneas $(n=25)$ rectilíneas y suavemente sinuosas y curvilíneas, dispuestas oblicuas hacia la derecha y tendentes a paralelizarse entre sí. En la mitad inferior se documentan dos líneas horizontales de diferente recorrido. 


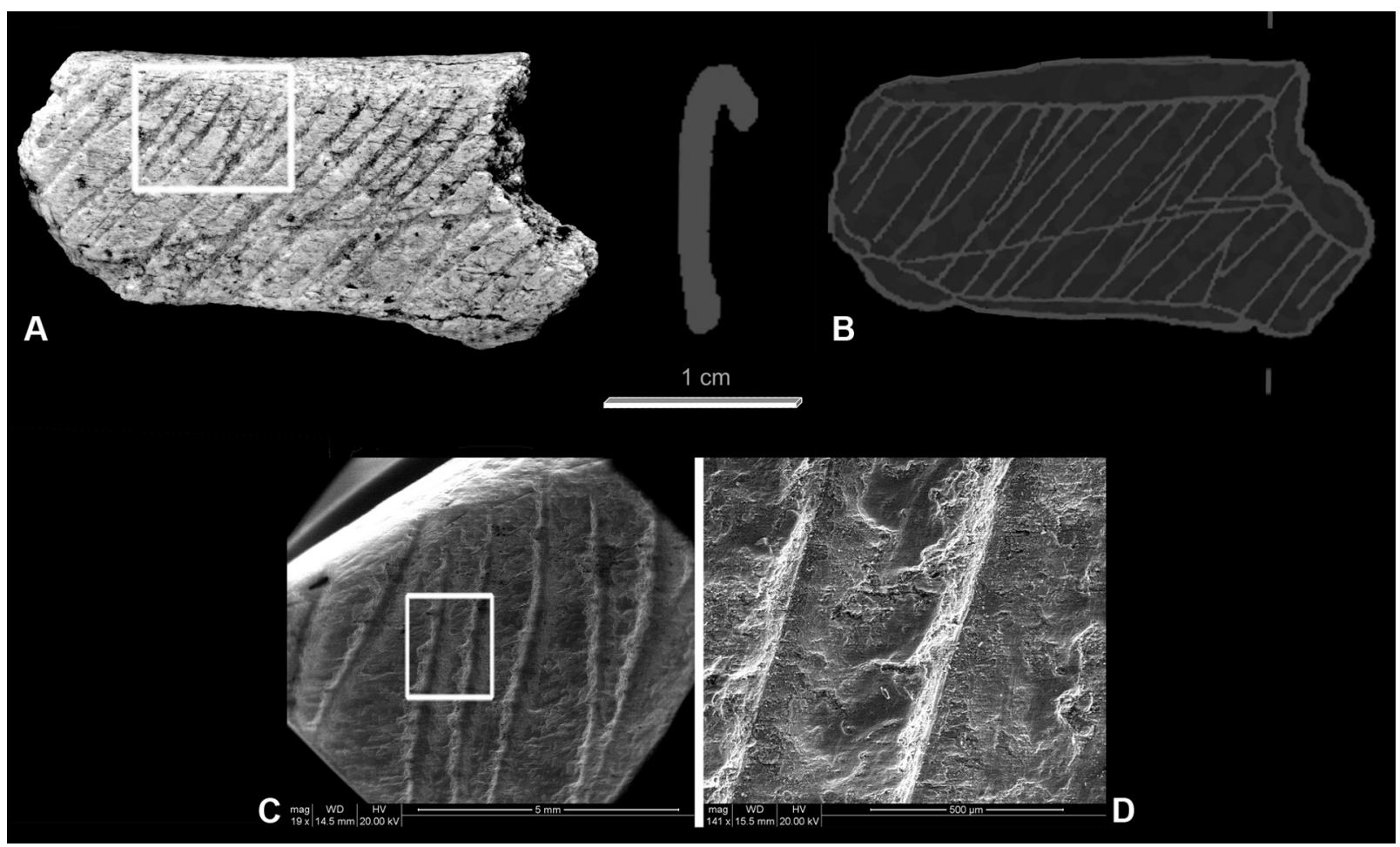

Fig. 2. Hueso recuperado en la Sala de las Chimeneas de la cueva de Maltravieso (Cáceres, España). A: fotografía; B: calco; C: microfotografía general de los surcos; D: detalle de surco grabado.

Para el estudio del proceso de ejecución de la decoración se utilizó una lupa binocular (Nikon SMZ 1500) y un Microscopio Electrónico de Barrido (ESEM FEI Quanta 600). Las condiciones de trabajo en el MEB fueron a bajo vacío y a $20 \mathrm{kV}$. La interpretación del proceso técnico (inicio y final de trazo, sentido y orden del trazado, etc.) se realizó considerando estigmas técnicos (estrías, morfologías, anchuras, superposiciones, etc.) obtenidos a partir de programas experimentales(1) (D’Errico 1994; Fritz 1999). Considerando las limitaciones de los procesos tafonómicos, se apuntan las siguientes consideraciones de orden técnico (Fig. 2C y D): a) la sección en ' $\mathrm{V}$ ' del surco permite considerar que las líneas fueron realizas con un instrumento de filo diedro; b) en las líneas de disposición oblicua, la mayor anchura de estas en su parte distal frente al desarrollo central y proximal que son más estrechos, la morfología roma en su parte distal y apuntada en la proximal, y los

(1) Cremades, M. 1989: Contribution à l'étude de l'art mobilier du Paléolithique supérieur du Bassin aquitain: techniques de gravure sur os et matériaux organiques. Tesis doctoral. Universidad de Bordeaux I. surcos parásitos en la parte proximal, indican que las líneas fueron realizadas de arriba hacia abajo; c) la línea de tendencia horizontal y de mayor recorrido fue trazada de derecha a izquierda; y d) muy probablemente, la línea horizontal se realizó con anterioridad a las verticales.

\section{DISCUSIÓN}

\subsection{El soporte decorado en el contexto peninsular y pirenaico}

Los soportes muebles con elementos gráficos en contextos superopaleolíticos peninsulares son poco frecuentes fuera del ámbito cantábrico. En el ámbito geográfico de la cueva de Maltravieso, el interior peninsular (Fig. 3), su presencia es muy escasa: El Caballón (Ibero 1923), La Hoz (Balbín et al. 1995), Villalba (Jimeno et al. 1990), Estebanvela (Cacho et al. 2007) y Jarama (Jordá y García 1989). En este contexto de baja densidad de arte mueble destaca la pieza de Maltravieso. Todos estos conjuntos presentan un componente

Trab. Prehist., 69, N. ${ }^{\text {2 }}$ 2, julio-diciembre 2012, pp. 349-356, ISSN: 0082-5638

doi: $10.3989 /$ tp.2012.12096 


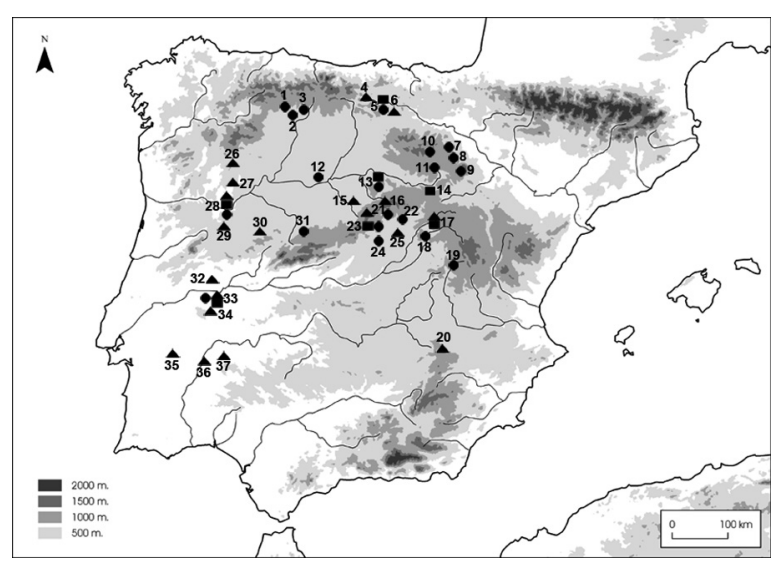

Fig. 3. Distribución de las ocupaciones humanas (círculo), arte mueble (cuadrado) y arte rupestre (triángulo) del interior peninsular. 1: La Cantera, 2: La Uña, 3: El Espertín, 4: Ojo Guareña, 5: El Caballón, 6: Penches, 7: Bolichera, 8: Peña del Diablo, 9: El Gato, 10: Villalba, 11: Peña del Manto, 12: El Palomar, 13: Estebanvela, 14: Villalba, 15: Domingo García, 16: La Griega, 17: La Hoz y Los Casares, 18: Buendía, 19:Verdelpino, 20: El Niño, 21: Los Enebrales, 22: Peña Capón, 23: Jarama, 24: El Sotillo, 25: El Turismo, El Cojo y El Reno, 26: Alto Sabor, 27: Ribeira da Sardinha, 28: Bajo Côa, 29: Alto Côa, 30: Siega Verde, 31: La Dehesa, 32: Ocreza, 33: Maltravieso, 34: Mina de Ibor, 35: Escoural, 36: Cheles y 37: Minerva.

gráfico figurativo. Pero en Estebanvela la decoración lineal es mayoritaria y diversa, y destacan los motivos de series de líneas paralelas ordenadas que se asocian a los bordes de los soportes pétreos y se distribuyen de manera afrontada.

Por el contrario, el fenómeno rupestre paleolítico está bien representado en estaciones al aire libre y en cavidades (Alcolea y Balbín 2003, 2006), correspondiendo a la zona extremeña (Collado 2009) el Molino Manzánez (Collado 2006), la Mina de Ibor (Ripoll y Collado 1997), el abrigo de Minerva (Collado 2003) y la propia cueva de Maltravieso. En territorio portugués se localiza la cueva de Escoural (Leujene 1996), donde algunas composiciones lineales se vinculan al motivo objeto de estudio. La contextualización estratigráfica y cronológica de la pieza de Maltravieso, junto al conjunto de Estebanvela datado entre $12440 \pm 50$ BP y $10640 \pm 60 \mathrm{BP}$, permiten sentar las bases para una futura caracterización del ciclo gráfico de este sector, ya para el resto de las piezas muebles no ha sido posible definirla.

El hueso de Maltravieso también reproduce un tipo decorativo lineal frecuente en el corpus mueble cantábrico (Barandiarán 1973, 1994; Corchón
1986, 2004; Fernández-Tresguerres 1994, 2004) desde el inicio del Paleolítico superior hasta momentos azilienses: en el Auriñaciense evolucionado de Rascaño; Gravetiense de El Pendo, Santimamiñe y Bolinkoba; Solutrense de Aitzbitarte, Altamira, El Pendo, Las Caldas y Cueto de la Mina; Magdaleniense inferior de Altamira, La Pasiega, El Castillo, El Juyo, Rascaño, Bolinkoba, Abauntz, El Cierro y Balmori; Magdaleniense medio de $\mathrm{La} \mathrm{Pa-}$ loma y Cueto de la Mina; Magdaleniense superior de Tito Bustillo, La Riera, El Castillo, Cueva Morín, La Paloma, Cueva Oscura de Ania, La Lloseta, El Pendo, El Valle, Urtiaga y Aitzbitarte: y Aziliense de La Paloma, Candamo y Ekain.

En el ámbito mediterráneo la composición grabada de Maltravieso corresponde al tipo 'haz de líneas paralelas aisladas y normales' individualizado en el yacimiento de El Parpalló (Villaverde 1994, 2005). Es uno de los motivos más frecuentes de la secuencia y que aparece desde el Solutrense inferior hasta el Magdaleniense superior.

En territorio francés se encuentran paralelos (Chollot 1980) en toda la secuencia supero-paleolítica: Auriñaciense (p.e Blanchard, La Ferrasie, Aurignac y Castanet), Gravetiense (p.e. Isturitz, Gargas y Saint-Jean-de-Verges), Solutrense (p.e. Le Placard, La Ruth y Tourtoirac) y Magdaleniense (p.e. Arudy, Le Placard, Laugerie-Haute, Mas d'Azil y Gourdan), reafirmando la amplia dispersión temporal del tema decorativo en el ámbito peninsular.

El carácter rectilíneo, seriado, regular, paralelo y organizado del soporte óseo de Maltravieso encuentra correspondencia en numerosos yacimientos, especialmente de la zona cantábrica y pirenaica, y con una menor dispersión en el área mediterránea e interior peninsular. Representa un tema que se documenta a lo largo de toda la secuencia del Paleolítico superior, y que incluso aparece en fases posteriores (Alday y García 1998; Martínez-Moreno et al. 2011). En esta cueva la correspondencia espacial entre los materiales datados del nivel A y la pieza ósea decorada certifican que esta se grabó entre hace 21758 y 20885 cal BP.

\subsection{El soporte decorado en el contexto de la cueva}

El arte rupestre de Maltravieso (Ripoll et al. 1999) presenta un pequeño conjunto de signos, entre los que se incluyen tres haces de líneas 
verticales que se asemejan al motivo mueble. Dos conjuntos, compuestos por 7 y 8 líneas rectilíneas, trazados en color marrón rojizo y asociados a una cabeza de caballo y una forma semicircular (Fig. 4), se localizan en la misma sala en la que se recuperó el soporte óseo y en el mismo panel (XIV), cuya implantación permite que sean fácilmente visualizadas.

El otro, de estructura más simple al estar compuesto por dos líneas negras, se localiza al inicio de la Sala, en el Corredor de la Serpiente (panel VIII), un espacio lateral y reservado, por lo que la observación de la grafía exige una detenida búsqueda o el conocimiento previo de su implantación. Estos espacios, que se sitúan cerca de la Sala de las Pinturas donde se encontraría la en-

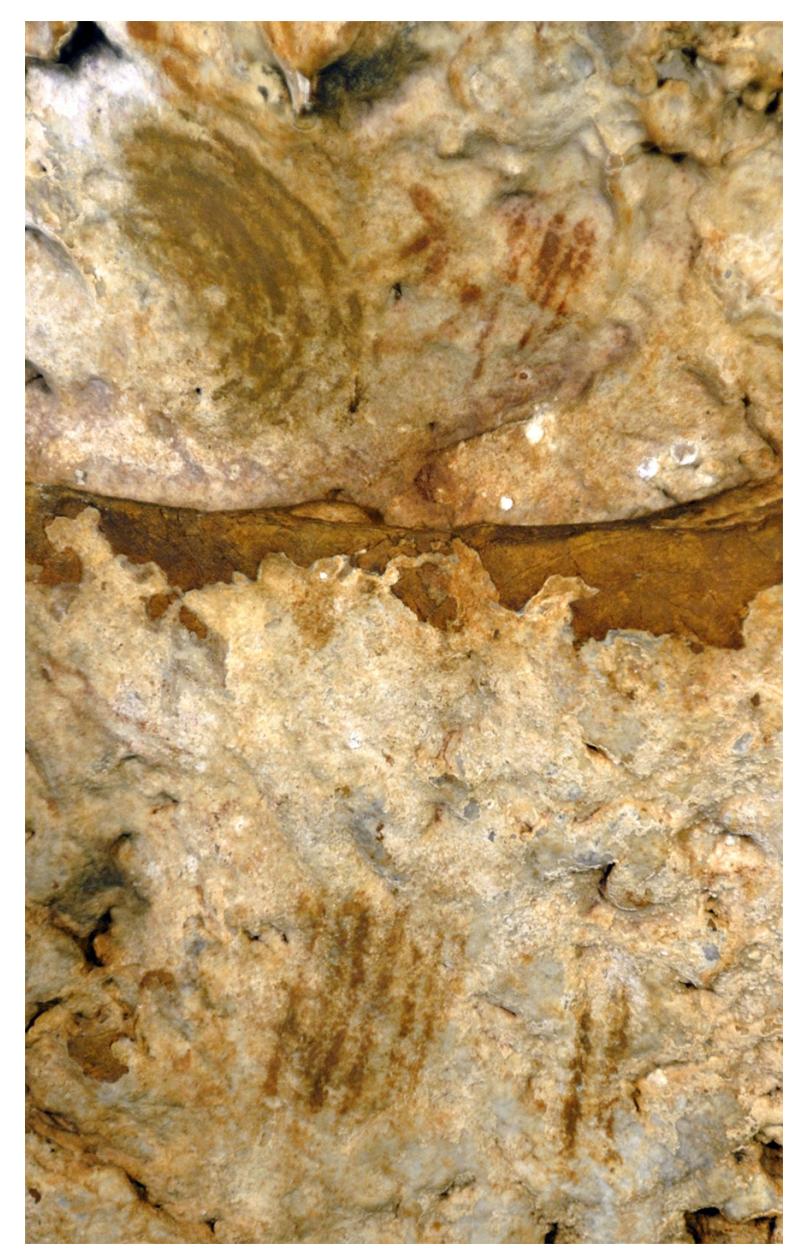

Fig. 4. Series de signos lineales rectilíneos y paralelos correspondientes al panel XIV del arte rupestre de la Sala de las Chimeneas de la cueva de Maltravieso (Cáceres, España). trada paleolítica(2), eran entornos de interior y oscuros. Las composiciones rupestres han sido atribuidas a un momento de transición entre el Solutrense final y el Magdaleniense inicial.

Las similitudes formales y compositivas, y la estrecha vinculación espacial entre formas rupestres y muebles, implica considerar como probable la hipótesis de una sincronía en la ejecución de grafismos y de que ambos soportes decorativos formaran parte de una misma estructura iconográfica y simbólica, como se ha documentado en cuevas cantábricas (Arias y Ontañón 2004).

\section{CONCLUSIÓN}

El fragmento de costilla con decoración lineal de la cueva de Maltravieso es un ejemplo de arte mueble del Paleolítico superior peninsular. Su recuperación en contexto estratigráfico, datado en 21758-20885 cal BP, representa una aportación para el conocimiento de la dispersión del grafismo paleolítico, completando un área geográfica (el sector extremeño) hasta ahora ausente de este tipo de soportes, frente a la presencia de conjuntos rupestres. La decoración corresponde a una serie de líneas rectilíneas, paralelas y regulares, realizadas a partir de trazos cortos y profundos ejecutados con un útil de filo diedro.

Por su temática y simplicidad compositiva muestra paralelos con yacimientos peninsulares y franceses, que abarcan una dilatada cronología a lo largo de todo el Paleolítico superior. La relación espacial y tipología entre el soporte mueble y al menos una parte del arte parietal de la propia cueva, permite avanzar en la contextualización cronológica del arte rupestre y considerar que ambas expresiones gráficas fueron realizadas dentro de un mismo ámbito ideológico.

\section{AGRADECIMIENTOS}

El trabajo ha sido realizado en el ámbito del proyecto 'Primeros Pobladores de Extremadura', dirigido por Eudald Carbonell Roura (Instituto Catalán de Paleoecología Humana y Evolución

(2) Mancha, E. 2011: Geología sedimentaria y lugares de ocupación prehistóricos de la Cueva de Maltravieso (Cáceres, España). Tesis doctoral. Universidad Rovira i Virgili.

Trab. Prehist., 69, N. ${ }^{\circ}$ 2, julio-diciembre 2012, pp. 349-356, ISSN: 0082-5638

doi: $10.3989 /$ tp.2012.12096 
Social) y uno de los autores (ACS) ARH es beneficiario del programa de ayudas FPI del MINECO (CGL2009-12703-C03-02). Las investigaciones en la Cueva de Maltravieso son subvencionadas por el Instituto Catalán de Paleoecología Humana y Evolución Social, la Consejería de Educación y Cultura del Gobierno de Extremadura, y los Ayuntamientos de Cáceres y Malpartida de Cáceres.

\section{BIBLIOGRAFÍA}

Alcolea, J. J. y Balbín, R. de 2003: “El arte rupestre paleolítico del interior peninsular. Elementos para el estudio de su variabilidad regional". En R. de Balbín y P. Bueno (eds.): El Arte Prehistórico desde los inicios del siglo XXI. Asociación Cultural Amigos de Ribadesella. Ribadesella: 223-253.

Alcolea, J. J. y Balbín, R. de 2006: Arte paleolitico al aire libre. El yacimiento rupestre de Siega Verde, Salamanca. Memoria Arqueología en Castilla y León, Junta de Castilla y León. Valladolid.

Alday, A. y García-Díez, M. 1998: “Evidencia gráfica mueble de cronología neolítica en el abrigo de Atxoste (Vírgala, Álava)". Veleia 15: 101-120.

Almagro-Basch, M. 1960: "Las pinturas rupestres cuaternarias de la cueva de Maltravieso, en Cáceres". Revista de Archivos, Bibliotecas y Museos LXVIII (2): 665-707.

Arias, P. y Ontañón, R. 2004: "El contexto del arte mobiliar paleolítico en la región Cantábrica”. En P. Arias y R. Ontañón (eds.): La materia del lenguaje prehistórico. Universidad de Cantabria. Santander: $37-52$.

Aubry, T. 2009: 200 séculos da história do Vale do Côa. Incursoes na vida cuotidiana dos caçadoresartistas do Paleolítico. Trabalhos de Arqueología 52, IGESPAR. Lisboa.

Aubry, T; Mangado, X.; Sellami, F. y Sampaio, J. D. 2002: "Open-air rock art. Territories and modes of exploitation during the Upper Paleolithic in the Côa Valley (Portugal)". Antiquity 76 (291): 62-76".

Aubry, T. y Sampaio, J. D. 2009: "Chronologie et context archéologique des gravures paléolithiques de plein air de la Vallée du Côa (Portugal)". En R. de Balbín (ed.): Arte paleolítico al aire libre en el Sur de Europa. Junta de Castilla y León. Valladolid: 211-224.

Balbín, R. de 2009: "El arte rupestre paleolítico al aire libre en la Península Ibérica". En R. de Balbín (ed.): Arte paleolítico al aire libre en el Sur de Europa. Junta de Castilla y León. Valladolid: 19-56.

Balbín, R. de; Alcolea, J. J. y Cruz, L. A. 1995: “Las placas decoradas de la Cueva de la $\mathrm{Hoz}$ (Sta. M. ${ }^{\mathrm{a}}$ del Espino, Guadalajara): un ejemplo de arte mobiliar paleolítico en la meseta castellana". I. ${ }^{\circ}$ Congresso de Arqueología Peninsular (Oporto 1993). Trabalhos de Antropología e Etnología XXXV. Sociedad Portuguesa de Antropología y Etnología. Oporto: 49-63.

Bañuls, S. y López-García, J. M. 2010: "Nota preliminar sobre el estudio de los roedores del Pleistoceno superior de la Sala de las Chimeneas de la Cueva de Maltravieso (Cáceres, Extremadura)". En M. Moreno-Azanza, I. Díez-Martínez, J. M. Gasca, M. Melero-Rubio y V. Sauqué (coords.): Actas del VIII encuentro de jóvenes investigadores en Paleontología (Enciso-La Rioja, 2009). Cidaris 30: 67-72.

Baptista, A. M. y Reis, M. 2009: "Prospecçao da arte rupestre no Vale do Côa e Alto Douro portugués: ponto da situaçao em julo de 2006". En R. de Balbín (ed.): Arte paleolítico al aire libre en el Sur de Europa. Junta de Castilla y León. Valladolid: 145-192.

Barandiarán, I. 1973: Arte mueble del Paleolítico cantábrico. Monografías Arqueológicas XIV. Universidad de Zaragoza. Zaragoza.

Barandiarán, I. 1994: “Arte mueble del Paleolítico cantábrico: una visión de síntesis en 1994". Complutum 5: 45-79.

Breuil, H. 1960: "Dècouverte d'una grotte ornée paléolithique dans la province de Cáceres (nord-ouest de l'Espagne)". Bulletin de la Société Préhistorique Française LVII: 155.

Cacho, C.; Ripoll, S. y Muñoz, F. J. 2007: La Peña de Estebanvela (Estebanvela-Ayllón, Segovia): grupos magdalenienses en el sur del Duero. Memoria Arqueología en Castilla y León, Junta de Castilla y León. Valladolid.

Callejo Serrano, C. 1958: La cueva prehistórica de Maltravieso, junto a Cáceres. Publicaciones de la Biblioteca Pública de Cáceres. Cáceres.

Canals, A.; Rodríguez-Hidalgo, A.; Peña, L.; Mancha, E.; García-Díez, M.; Bañuls, S.; Euba, I.; LópezGarcía, J. M.; Barrero, N.; Bermejo, L.; García, F. J.; Mejías, D.; Modesto, M.; Morcillo, A.; Aranda, V. y Carbonell, E. 2010: "Nuevas aportaciones al Paleolítico superior del suroeste peninsular: la cueva de Maltravieso, más allá del santuario extremeño de las manos". En X. Mangado (ed.): El Paleolítico superior peninsular: Novedades del S. XXI. Homenaje al profesor Javier Fortea. Monografías del Seminari d'Estudis i Recerques Prehistòriques 8. Barcelona: 157-176.

Chollot Varagnac, M. 1980. Les origines du graphisme symbolique. Essai d'analyse des écritures primitives en Préhistoire. Fondation Singer-Polignac. Paris.

Collado, H. 2003: "Nuevas representaciones de arte paleolítico en Extremadura". CAEAP veinticinco sobre el Patrimonio Cultural de Cantabria. Ayuntamiento de Camargo. Santander: 111-120. 
Collado, H. 2006: Arte rupestre en la cuenca del Guadiana: el conjunto de grabados del Molino Manzánez (Alconchel-Cheles). Memórias d'Odian, Estudios Arqueológicos de Alqueva 4. EDIA. Beja.

Collado, H. 2009: "Arte rupestre prehistórico en Extremadura: 1997-2006”. En R. de Balbín (ed.): Arte paleolítico al aire libre en el Sur de Europa. Junta de Castilla y León. Valladolid: 287-322.

Corchón, M. ${ }^{a}$ S. 1986: El arte mueble Paleolítico cantábrico: contexto y análisis interno. Centro de Investigación y Museo de Altamira, monografías 16. Ministerio de Cultura. Madrid.

Corchón, M. a S. 2002: "El Tardiglaciar y la transición al Postglaciar en la Meseta norte española: una revisión de síntesis". Zephyrvs 55: 85-142.

Corchón, M. ${ }^{a}$ S. 2004. "El arte mueble paleolítico en la Cornisa Cantábrica y su prolongación en el Epipaleolítico". En M. A. Fano (coord.): Las Sociedades del Paleolítico en la Región Cantábrica. Kobie anejo 8. Diputación Foral de Vizcaya. Bilbao: 425474.

Delibes, G y Díez, F. 2006: El Paleolítico superior en la Meseta norte española. Studia Archaeologica 94. Universidad de Valladolid. Valladolid.

d'Errico, F. 1994: L'art gravé Azilien. De la technique à la signification. XXXIème à Gallia Préhistorique. CNRS. Paris.

Fernández Tresguerres, J. A. 1994: "El arte aziliense”. Complutum 5: 81-95.

Fernández Tresguerres, J. A. 2004. "El final del Paleolítico en los espacios cantábricos: el Aziliense". En M. A. Fano (coord.): Las Sociedades del Paleolítico en la Región Cantábrica. Kobie anejo 8. Diputación Foral de Vizcaya. Bilbao: 309-336.

Fritz, C. 1999. La gravure dans l'art mobilier magdalénien, du geste à la représentation. Contribution de l'analyse microscopique. Documents d'Archéologie Française 75, Maison des Sciences de 1'Homme. Paris.

García-Díez, M y Aubry, T. 2002: "Grafismo mueble en el valle de Côa (Vila Nova de Foz Côa, Portugal): la estación arqueológica de Fariseu". Zephyrvs LV: $157-182$.

Ibero, J. M. 1923: "El Paleolítico en Oña y sus alrededores (Burgos)". Razón y Fe 67: 171-194.

Jimeno, A.; Fernández Moreno, J. J.; Gómez Barrera, J. A. y Galindo, M. P. 1990: "Arte paleolítico en la provincia de Soria". Numantia 3: 9-50.

Jordá Cerdá, F. 1971: "Sobre la edad de las pinturas de la cueva de Maltravieso (Cáceres)". En A. Beltran (ed.): Actas del XI. ${ }^{\circ}$ Congreso Nacional de Arqueología (Mérida, 1968): 139-153. Zaragoza.

Jordá Cerdá, F. y Sanchidrián, J. L. 1992: La Cueva de Maltravieso. Guía del visitante. Editorial Regional Extremeña. Mérida.
Jordá Pardo, J. F. y García Valero, M. A. 1989: "Las representaciones de glotón (Gula gula) en el Arte Paleolítico pirenaico y un nuevo hallazgo de arte mueble en el Alto Valle del Jarama (Guadalajara)". Espacio, Tiempo y Forma serie I (2): 89-105.

Leujene, M. 1996. "L'art parietal". En M. Otte y A. C. Silva (dirs.): Recherches préhistoriques à la grotte d'Escoural, Portugal. Etudes et Recherches Archeologiques de l'Universite de Liege 65. Universite de Liege. Liège: 135-240.

Mangado, X 2010: El Paleolítico superior peninsular. Novedades del siglo XXI. Homenaje al profesor Javier Fortea. Monografías del Seminari d'Estudis i Recerques Prehistòriques 8. Universitad de Barcelona. Barcelona.

Martínez-Moreno, J.; Villaverde, V. y Mora, R. 2011: "La placa grabada de Balma Guilanyà (Prepirineo de Lleida) y las manifestaciones artísticas del Mesolítico de la Península Ibérica". Trabajos de Prehistoria 68 (1): 159-173.

Ripoll, S. y Collado, H. 1997: "La découverte de nouvelles représentations Paléolitiques en Extremadura: la grotte de la Mina de Ibor". International Newsletters on Rock Art 17: 8-11.

Ripoll, S.; Ripoll, E. y Collado, H. 1999: Maltravieso. El santuario extremeño de las manos. Memoria I del Museo de Cáceres. Mérida.

Rodríguez-Hidalgo, A. J.; Canals A.; Saladié, P. y Carbonell, E. 2010a: "Los cubiles de Crocuta crocuta como contenedores de información paleoecológica. El caso de la Sala de los Huesos. Cueva de Maltravieso". Zona Arqueológica 11: 256-249.

Rodríguez-Hidalgo, A.; Canals, A.; Saladie, P.; García, A. B. y García-Díez, M. 2010b: "Upper Palaeolithic ornaments seashells from Sala de las Chimeneas, Maltravieso cave, Cáceres (Spain)". Munibe 61: 35-44.

Rodríguez-Hidalgo, A.; Saladie, P. y Canals, A. 2011: "Following the white rabbit: a case of a small game procurement site in the Upper Palaeolithic (Sala de las Chimeneas, Maltravieso cave, Spain)". International Journal of Osteoarchaeology. doi: 10.1002/ oa. 1238

Simón, M. ${ }^{\text {a }}$ D.; Cortés, M. y Bicho, N. 2012: "Primeras evidencias de arte mueble paleolítico en el sur de Portugal". Trabajos de Prehistoria 69 (1): 7-20.

Villaverde, V. 1994: Arte paleolítico de la Cova del Parpalló. Estudio de la colección de plaquetas y cantos con grabados y pinturas. Diputación de Valencia. Valencia.

Villaverde, V. 2005: “Arte paleolítico de la región mediterránea de la Península Ibérica: de la cueva de La Pileta a la Cova de les Meravelles". En M. Hernández y J. Soler (eds.): Actas del Congreso de arte rupestre en la España mediterránea (Alicante 2004): 17-44. Alicante. 